\title{
Mean-field dynamics of a two-mode Bose-Einstein condensate subject to noise and dissipation
}

\author{
F Trimborn ${ }^{1}$, D Witthaut ${ }^{2}$ and S Wimberger ${ }^{3}$ \\ ${ }^{1}$ Institut für mathematische Physik, TU Braunschweig, D-38106 Braunschweig, \\ Germany \\ ${ }^{2}$ QUANTOP, Niels Bohr Institute, University of Copenhagen, DK-2100 \\ Copenhagen, Denmark \\ ${ }^{3}$ Institut für theoretische Physik, Universität Heidelberg, D-69120, Heidelberg, \\ Germany \\ E-mail: dirk.witthaut@nbi.dk
}

\begin{abstract}
We discuss the dynamics of an open two-mode Bose-Hubbard system subject to phase noise and particle dissipation. Starting from the full many-body dynamics described by a master equation the mean-field limit is derived resulting in an effective non-hermitian (discrete) Gross-Pitaevskii equation which has been introduced only phenomenologically up to now. The familiar mean-field phase space structure is substantially altered by the dissipation. Especially the character of the fixed points shows an abrupt transition from elliptic or hyperbolic to attractiv or repulsive, respectively. This reflects the metastable behaviour of the corresponding many-body system which surprisingly also leads to a significant increase of the purity of the condensate. A comparison of the mean-field approximation to simulations of the full master equation using the Monte Carlo wave function method shows an excellent agreement for wide parameter ranges.
\end{abstract}

PACS numbers: 03.75.Lm, 03.75.Gg, 03.65.Yz 
The physics of ultracold atoms in optical lattices has made an enormous progress in the last decade, as it is an excellent model system for a variety of fields such as nonlinear dynamics or condensed matter physics [1,2]. Although this seems to be an inherent many-particle problem, the dynamics of the macroscopic wave function is remarkably well reproduced by the (discrete) Gross-Pitaevskii equation (GPE) if the systems undergoes a Bose-Einstein condensation [3]. Recently there has been an increased theoretical [4-8] as well as experimental [9] interest in the dynamics of these systems coupled to the environment. In particular, the effects of particle loss have been discussed in several theoretical articles resorting to an effective non-hermitian meanfield description introduced phenomenologically to analyse resonances, transport and localization effects [10-16].

In this communication we want to illuminate the origin and give a convincing motivation of this approach. Starting from a master equation describing the full manybody dynamics including phase noise and particle loss we derive a generalized, nonhermitian Gross-Pitaevskii equation. Due to the decay the structure of the resulting dynamics abruptly changes introducing repulsive and attractive fixed points. Unlike the dissipation-free case there are no longer oscillations around the fixed points, such that irreversible transitions between the former self-trapping fixed points are possible. This reflects the metastable behaviour of the open many-particle system and gives rise to a significant purification of the BEC by the dissipation which will be explained here. The validity of the presented approximation is tested by a comparison to full quantum manybody calculations, showing that the mean-field approximation provides an excellent description of the system. To integrate the effective description by a non-hermitian GPE into well-known concepts from the theory of open quantum systems, we discuss their relation to the quantum jump approach $[17,18]$, demonstrating that they provide a well-suited tool to analyse the short- as well as the long-time behaviour of the open many-particle system.

In particular, we consider the dynamics of ultracold atoms in an open double-well trap which is not only an extremely popular model system, but also has several recent experimental realisations [19-22]. The unitary part of the dynamics is described by the Bose-Hubbard type Hamiltonian

$$
\hat{H}=-J\left(\hat{a}_{1}^{\dagger} \hat{a}_{2}+\hat{a}_{2}^{\dagger} \hat{a}_{1}\right)+\epsilon_{1} \hat{n}_{1}+\epsilon_{2} \hat{n}_{2}+\frac{U}{2}\left(\hat{a}_{1}^{\dagger 2} \hat{a}_{1}^{2}+\hat{a}_{2}^{\dagger 2} \hat{a}_{2}^{2}\right),
$$

where $\hat{a}_{j}$ and $\hat{a}_{j}^{\dagger}$ are the bosonic annihilation and creation operators in mode $j$ and $\hat{n}_{j}=\hat{a}_{j}^{\dagger} \hat{a}_{j}$ is the correponding number operator. We set $\hbar=1$, thus measuring all energies in frequency units. In order to analyze the dynamics in the Bloch-representation we transform the Hamiltonian using the collective operators

$$
\begin{gathered}
\hat{L}_{x}=\frac{1}{2}\left(\hat{a}_{1}^{\dagger} \hat{a}_{2}+\hat{a}_{2}^{\dagger} \hat{a}_{1}\right), \quad \hat{L}_{y}=\frac{\mathrm{i}}{2}\left(\hat{a}_{1}^{\dagger} \hat{a}_{2}-\hat{a}_{2}^{\dagger} \hat{a}_{1}\right), \\
\hat{L}_{z}=\frac{1}{2}\left(\hat{a}_{2}^{\dagger} \hat{a}_{2}-\hat{a}_{1}^{\dagger} \hat{a}_{1}\right),
\end{gathered}
$$

which form an angular momentum algebra $s u(2)$ with rotational quantum number 
$N / 2[6,23-25]$. With these definitions the Hamiltonian (1) can be rewritten as

$$
\hat{H}=-2 J \hat{L}_{x}+2 \epsilon \hat{L}_{z}+U \hat{L}_{z}^{2}
$$

with $2 \epsilon=\epsilon_{1}-\epsilon_{2}$ up to terms which only depend on the total number of atoms. The macroscopic dynamics of the atomic cloud is well described within a mean-field approximation, only considering the expectation values of the angular momentum operators $\ell_{j}=\left\langle\hat{L}_{j}\right\rangle$ and the particle number $n=\left\langle\hat{n}_{1}+\hat{n}_{2}\right\rangle[6,23,24]$.

Here we consider the dissipative extension of this system. The main source of decoherence in current experiments is phase noise due to elastic collision with atoms in the thermal cloud $[4,5]$ which effectively heats the system, leaving the particle number invariant. Only recently, methods to tame this source of decoherence were discussed in $[30,31]$. In this communication we focus on the effects of particle loss at constant rates $\gamma_{a j}$ in the two wells $j=1,2$. Such a loss is not only of fundamental interest but can be adapted in current experiments without greater difficulties by removing atoms with a focused resonant laser beam or by inducing a radio frequency transition to an untrapped internal state [26]. All parameters used here lie in realistic ranges for ongoing experiments $[19,20]$.

The master equation description including both, phase noise and particle loss, is well established [27] and routinely used in the context of photon fields. Thus we consider the dynamics generated by the master equation

$$
\begin{array}{r}
\dot{\hat{\rho}}=-\mathrm{i}[\hat{H}, \hat{\rho}]-\frac{\gamma_{p}}{2} \sum_{j=1,2}\left(\hat{n}_{j}^{2} \hat{\rho}+\hat{\rho} \hat{n}_{j}^{2}-2 \hat{n}_{j} \hat{\rho} \hat{n}_{j}\right) \\
-\frac{1}{2} \sum_{j=1,2} \gamma_{a j}\left(\hat{a}_{j}^{\dagger} \hat{a}_{j} \hat{\rho}+\hat{\rho} \hat{a}_{j}^{\dagger} \hat{a}_{j}-2 \hat{a}_{j} \hat{\rho} \hat{a}_{j}^{\dagger}\right) .
\end{array}
$$

The evolution equations for the expectation values of the angular momentum operators (2) can be calculated starting from the Master equation via $\dot{\ell}_{j}=\operatorname{tr}\left(\hat{L}_{j} \dot{\hat{\rho}}\right)$ with $j=x, y, z$. This yields the exact result

$$
\begin{aligned}
& \dot{\ell}_{x}=-2 \epsilon \ell_{y}-2 U\left(\ell_{y} \ell_{z}+\Delta_{y z}\right)-T_{2}^{-1} \ell_{x}, \\
& \dot{\ell}_{y}=2 J \ell_{z}+2 \epsilon \ell_{x}+2 U\left(\ell_{x} \ell_{z}+J_{x z}\right)-T_{2}^{-1} \ell_{y}, \\
& \dot{\ell}_{z}=-2 J \ell_{y}-T_{1}^{-1} \ell_{z}-T_{1}^{-1} f_{a} n / 2, \\
& \dot{n}=-T_{1}^{-1} n-2 T_{1}^{-1} f_{a} \ell_{z},
\end{aligned}
$$

where we have defined the transversal $T_{1}^{-1}$ and longitudinal $T_{2}^{-1}$ damping rates by

$$
T_{1}^{-1}=\left(\gamma_{a 1}+\gamma_{a 2}\right) / 2 \text { and } T_{2}^{-1}=\gamma_{p}+T_{1}^{-1}
$$

and the asymmetry of the loss rates by $f_{a}=\left(\gamma_{a 2}-\gamma_{a 1}\right) /\left(\gamma_{a 1}+\gamma_{a 2}\right)$. In the noninteracting case $U=0$, these equations of motion ressemble the Bloch equations in nuclear magnetic resonance [28], except for the fact that the 'equilibrium' value of the population imbalance $\ell_{z}$ is given by $-f_{a} n / 2$ and therefore depends on the decreasing expectation value of the total particle number $n$.

The exact equations of motion (5) still include the covariances $\Delta_{j k}=\left\langle\hat{L}_{j} \hat{L}_{k}+\right.$ $\left.\hat{L}_{k} \hat{L}_{j}\right\rangle / 2-\left\langle\hat{L}_{j}\right\rangle\left\langle\hat{L}_{k}\right\rangle$. The approximation of second order moments by products of 


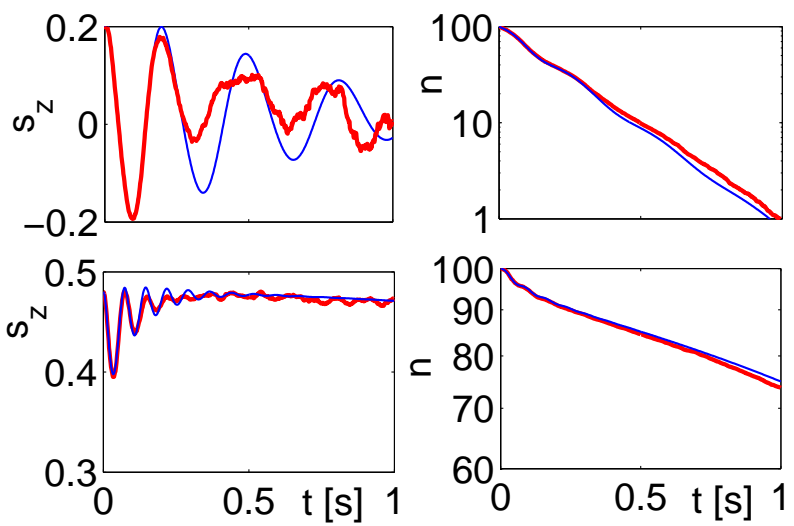

Figure 1. Comparison of the mean-field approximation (thin blue line) with the full many-particle dynamics calculated with the MCWF method (thick red line) for $J=10 \mathrm{~s}^{-1}, U=1 \mathrm{~s}^{-1}, \gamma_{p}=3 \mathrm{~s}^{-1}$ and $\gamma_{a 2}=5 \mathrm{~s}^{-1}$. The initial state was assumed to be a pure BEC (i.e. a product state) with $\mathbf{s}=(0.46,0,0.2)$ (a) and $\mathbf{s}=(0.14,0,0.48)(\mathrm{b})$, respectively, and $n=100$ particles.

expectation values, such that $\Delta_{j k} \approx 0$ yields the well-known mean-field description. This truncation is valid in the macroscopic limit, since the covarinces vanish as $1 / n$ if the many-particle quantum state is close to a pure BEC. Here and in the following we depict the rescaled variables $s_{j}=\ell_{j} / n$, thus renormalizing to separate the decay of the particle number $n$ from the internal dynamics.

The benefit of the mean-field approximation is illustrated in figure 1, where it is compared to the full many-particle quantum dynamics calculated with the Monte Carlo wave function (MCWF) method [17, 18]. The trajectory in figure 1 (a) was launched at $\mathbf{s}=(0.46,0,0.2)$ with a moderate population imbalance, thus performing Josephson oscillations [19]. The amplitude is damped because of the phase noise, while the oscillation period increases as the effective macroscopic interaction strength $g(t):=U n(t)$ decreases. The decay of the particle number $n(t)$ is also strongly modulated by the oscillations of the population imbalance. The trajectory in figure 1(b) was launched at $\mathbf{s}=(0.14,0,0.48)$ in the self-trapping region. The residual oscillations are rapidly damped out and the system relaxes to a quasi-steady state on the shown time scale. The particle number decreases slowly and non-exponentially, since the condensate is mostly localized in the non-decaying potential well, cf. also [15]. All these features of the dynamics are well reproduced by the mean-field description, and the decay of the particle number is accurately predicted. Strong deviations are only expected in the vicinity of unstable fixed points of the mean-field dynamics, which can be nearly cured within the framework of phase space distributions [25].

In order to explore the genuine effects of particle loss, phase noise is neglected $\left(\gamma_{p}=0\right)$ in the following. In this case the dynamics can be further simplified and one can easily show that the particle number coincides with the magnitude of the Bloch vector $\sqrt{\ell_{x}^{2}+\ell_{y}^{2}+\ell_{z}^{2}}=n / 2$, which we can use to reformulate the mean-field dynamics 

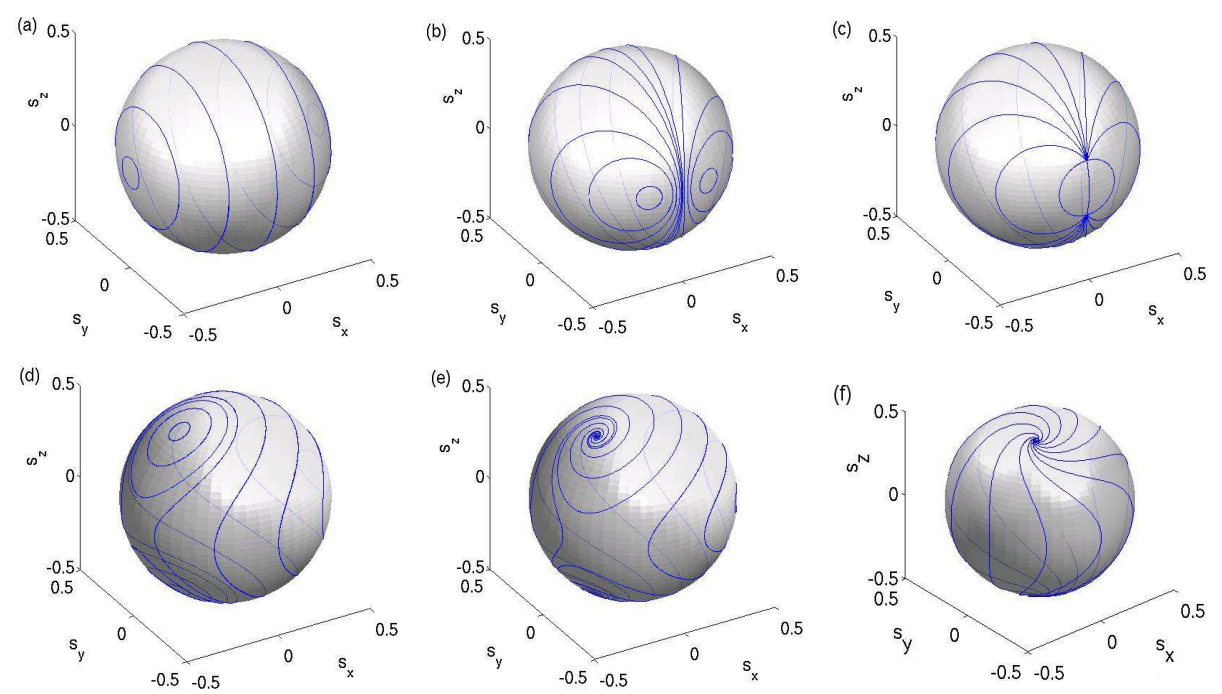

Figure 2. Mean-field dynamics for the non-interacting case $g=0$ (upper row) and for a fixed interaction strength $g=4 \mathrm{~s}^{-1}$ (lower row) in dependence of the decay rate $\left(\gamma=0\right.$ for (a) and (d), $\gamma=1.9 \mathrm{~s}^{-1}$ for $(\mathrm{b}), \gamma=2.1 \mathrm{~s}^{-1}$ for $(\mathrm{c}), \gamma=1 \mathrm{~s}^{-1}$ for (e) and $\gamma=4 \mathrm{~s}^{-1}$ for (f) $)$ - for all figures holds $J=1 \mathrm{~s}^{-1}$ and $\epsilon=0$.

by an effective non-hermitian Gross-Pitaevskii equation

$$
\mathrm{i} \frac{\mathrm{d}}{\mathrm{d} t}\left(\begin{array}{c}
\psi_{1} \\
\psi_{2}
\end{array}\right)=\left(\begin{array}{cc}
\tilde{\epsilon}_{1}+U\left|\psi_{1}\right|^{2} & -J \\
-J & \tilde{\epsilon}_{2}+U\left|\psi_{2}\right|^{2}
\end{array}\right)\left(\begin{array}{c}
\psi_{1} \\
\psi_{2}
\end{array}\right)
$$

with complex on-site energies $\tilde{\epsilon}_{j}=\epsilon_{j}-i \gamma_{a j} / 2$. The equivalence to the Bloch vector description is established via the identification

$$
\begin{gathered}
\ell_{x}=\frac{1}{2}\left(\psi_{1}^{*} \psi_{2}+\psi_{2}^{*} \psi_{1}\right), \quad \ell_{y}=\frac{1}{2 \mathrm{i}}\left(\psi_{2}^{*} \psi_{1}-\psi_{1}^{*} \psi_{2}\right), \\
\ell_{z}=\frac{1}{2}\left(\left|\psi_{2}\right|^{2}-\left|\psi_{1}\right|^{2}\right)
\end{gathered}
$$

and $n=\left|\psi_{1}\right|^{2}+\left|\psi_{2}\right|^{2}$. In this effective description a loss of particles is represented by a loss of normalization.

We now consider the dynamics for a fixed value of the macroscopic interaction strength $g=U n=$ const. in the special case $\gamma_{a 1}=: \gamma$ and $\gamma_{a 2}=0$. Even though the restriction to a fixed interaction constant seems to be artificial, it reveals the effects of the particle loss on the structure of the mean-field phase space and especially the character of the fixed points most clearly. Moreover, the dynamics under a fixed interaction constant correspond to the periods of constant particle number between two loss processes in the quantum jumps picture $[17,18]$. Therefore this treatment provides a well-suited description of the short- as well as the long-time behaviour. Note that the more general case $\gamma_{a 1} \neq 0$ and $\gamma_{a 2} \neq 0$ does not lead to a fundamentally different dynamical behaviour since only the difference of the decay rates influences the internal dynamics. However, the expectation value of the particle number $n$ and thereby also the effective interaction strength $g(t)$ decrease faster. 
The resulting dynamics of the Bloch vector is illustrated in figure 2, The upper row (a-c) shows the phase space for the linear case, $U=0$, where the mean-field approximation is exact. Without loss one recovers the famous Josephson oscillations (a). An analysis of the fixed points for the dissipative dynamics shows the emergence of two regimes depending on the amplitude of the loss rate. For weak losses, $|\gamma| \leq 2 J$, the fixed points are given by

$$
\mathbf{s}_{ \pm}^{J}=\left(\begin{array}{c} 
\pm\left[\frac{1}{4}-\left(\frac{\gamma}{4 J}\right)^{2}\right]^{\frac{1}{2}} \\
-\frac{\gamma}{4 J} \\
0
\end{array}\right)
$$

While the fixed points remain elliptic and the population is still equally distributed, the fixed points are no longer symmetric, since the relative phase between them decreases (b). This behaviour can be qualitatively understood within the analogy to Josephson junctions: The weak decay induces an assymetry between the wells leading to to a continuous particle stream to the first well. At the fixed points this effect is compensated by the Josephson current $I_{J} \propto J s_{y}$ requiring $s_{y} \neq 0$.

For stronger decay rates, $|\gamma| \geq 2 J$, the two fixed points are given by

$$
\mathbf{s}_{ \pm}^{D}=\left(\begin{array}{c}
0 \\
-\frac{J}{\gamma} \\
\pm\left[\frac{1}{4}-\left(\frac{J^{2}}{\gamma^{2}}\right)\right]^{\frac{1}{2}}
\end{array}\right) .
$$

Above the critical value $|\gamma|=2 J$ the character of the two fixed points changes abruptly from elliptic into an attractive and a repulsive one as shown in figure 2(c). The maximal Josephson current is no longer sufficient to compensate the current induced by the decay leading to a population excess in the non-decaying site. This explains the population imbalance in the fixed points which increases with ascending decay rates.

In the strongly interacting case without dissipation one observes the splitting of one of the elliptic fixed points into two novel elliptic and one hyperbolic fixed point - this is the famous self-trapping effect $[19,23,24]$. The critical interaction strength for the occurence of this bifurcation is lowered in the presence of dissipation to $g^{2}=U^{2} n^{2} \geq 4 J^{2}-\gamma^{2}$. In the subcritical regime for $\gamma<2 J$ and $U n \leq 4 J^{2}-\gamma^{2}$, we find oscillations around the same fixed points $s_{ \pm}^{J}$ as in the non-interacting, but dissipative case (9). However, these are now distorted (not shown in the figure). In the overcritical regime $g=U n>\sqrt{4 J^{2}+\gamma^{2}}$ and for a weak decay $\gamma<2 J$ one rediscovers a generalized self-trapping effect. As a result of the dissipative process, one elliptic fixed point now bifurcates into an attractive and a repulsive fixed point (in contrast to the two elliptic ones) and one hyperbolic one (cf. figure 2 (e)). The novel fixed points are located at:

$$
\mathbf{s}_{ \pm}^{\pi}=\frac{1}{\gamma^{2}+g^{2}}\left(\begin{array}{c}
-g J \\
-\gamma J \\
\pm \sqrt{\left(\gamma^{2}+g^{2}\right)\left(\frac{\gamma^{2}+g^{2}}{4}-J^{2}\right)}
\end{array}\right) .
$$



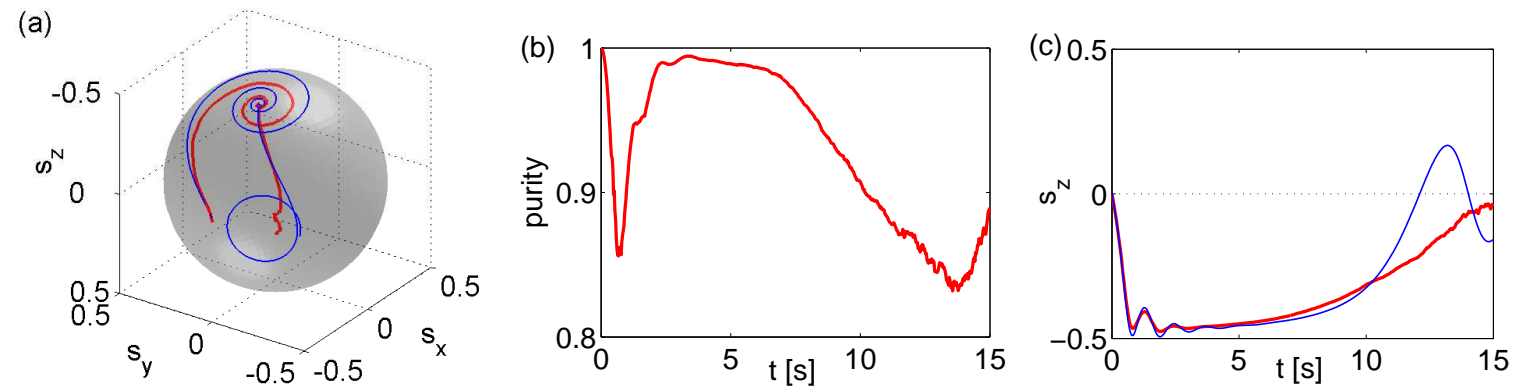

Figure 3. Comparison of the many-particle dynamics (thick red line) to the meanfield approximation (thin blues line) for an initially pure BEC. with $\mathbf{s}=(-0.5,0,0)$ and $n(0)=200$ particles: Dynamics of the Bloch vector $\mathbf{s}(\mathrm{a})$, evolution of the purity of the BEC (b) and evolution of the population imbalance $s_{z}$ (c). The system initially relaxes to a non-linear quasi-steady state with a purity of almost one which is then lost as $n(t)$ decreases. Parameters are chosen as $J=1 \mathrm{~s}^{-1}, U n(0)=10 \mathrm{~s}^{-1}, T_{1}=1 \mathrm{~s}$ and $f_{a}=1$.

For stronger decay rates, $\gamma \geq 2 J$, the hyperbolic and the elliptic fixed point $s_{ \pm}^{J}$ (9) meet and annihilate themselves as illustrated in figure 2 (f). Their disappearence is accompanied by the complete disintegration of periodic orbits.

Let us finally discuss the implication of this phase space structure. We especially focus on the emergence of the attractive fixed point since it is stable and therefore strongly influences the many-body quantum dynamics. Figure 3 shows the dynamics of the rescaled Bloch vector $\mathbf{s}$ comparing results of a MCWF simulation (solid red line) to the mean-field approximation (thin blue line). The given parameters correspond to the situations illustrated in figure 2 (b) or (e), respectively, depending on the value of the macroscopic interaction strength $g(t)=U n(t)$. The Bloch vector first relaxes to the attractive fixed point illustrated in figure 2 (e). The contraction of the mean-field trajectories to the attractive fixed point manifests itself by a convergence towards a pure BEC, which is the state of tightest localization in phase space [25]. This is illustrated in figure 3 (b) where we have plottet the purity $\mathcal{P}:=2 \operatorname{tr}\left(\rho_{\text {red }}^{2}\right)-1$ of the reduced single particle density matrix $\rho_{\text {red }}, \mathcal{P}=1$ indicating a pure BEC [6]. However, the attractive fixed point is lost as $g(t)=U n(t)$ decreases, and thus the Bloch vector departs again. This behaviour is very well prediced by the mean-field approximation already for the modest atom number in the simulation. The mean-field trajectory then convergences to the limit cycle shown in figure 2 (b). However, as the atoms are so rapidly lost nearly no particles remain to follow the limit cycle predicted by mean-field theory. This transition effect between different fixed points is closely related to the quantum state diffusion in and out of a metastable state, which can be observed in optical bistability (see, e.g., [32]). Note however, that the system considered here irretrievably departs from the metastable self-trapping state because the fixed point is lost as $n(t)$ decreases.

In summary, we have derived a mean-field approximation for a dissipative twomode BEC, starting from the full many-body dynamics described by a master equation including phase noise and particle losses. This treatment puts the so 
far phenomenological description of open systems via non-hermitian Gross-Pitaevskii equations on a firm footing and paves the way for a variety of future applications in particular because the extension to an arbitrary number of modes is straightforward.

An analysis of the resulting equations for a fixed interaction contant $g$ shows that not only the critical value for the self-trapping bifucation is lowered but also the character of the fixed points abruptly changes to attractive and repulsive, such that one of them becomes unstable. Taking into account the decline of the interaction constant $g(t)$ due to the particle losses, the system initially converges to the attractive fixed point, but then suddenly jumps to a Josephson oscillation as soon as $g(t)$ falls below the critical value. This effect is understood as a manifestation of the metastable behaviour of the many-particle system and leads to a significant increase of the purity of the quantum state compared to the dissipation-free case.

The comparison to numerical results for the many-particle system obtained via

the Monte Carlo Wave function method shows that the approach presented here is an excellent approximation to the full many-body dynamics already for a modest initial number of atoms. Thus it provides an excellent basis for a further analysis of the interplay between dissipation and interaction [31]. Likewise the embedding into the more general concept of mean-field description [33] will be subject to future research.

\section{Acknowledgments}

This work has been supported by the German Research Foundation (DFG) through the research fellowship programm (grant number WI 3415/1) and the Heidelberg Graduate School of Fundamental Physics (grant number GSC 129/1), as well as the Studienstiftung des deutschen Volkes. We thank H J Korsch, E M Graefe and A Niederle for stimulating discussions.

\section{References}

[1] Morsch O and Oberthaler M 2006 Rev. Mod. Phys. 78179

[2] Madroñero J et al. 2006 Adv. At. Mol. Opt. Phys. 5333 (ed M Scully and G Rempe)

[3] Pitaevskii L and Stringari S 2003 Bose-Einstein Condensation (Oxford University Press).

[4] Anglin J R 1997 Phys. Rev. Lett. 796

[5] Ruostekoski J and Walls D F 1998 Phys. Rev. A 58 R50

[6] Vardi A and Anglin J R 2001 Phys. Rev. Lett. 86 568; Anglin J R and Vardi A 2001 Phys. Rev. A 64013605

[7] Ponomarev A V, Madroñero J, Kolovsky A R and Buchleitner A 2006 Phys. Rev. Lett. 96050404

[8] Wang W, Fu L B and Yi X X 2007 Phys. Rev. A 75045601

[9] Syassen N, Bauer D M, Lettner M, Volz T, Dietze D, Garcia-Ripoll D D, Cirac J I, Rempe G and Dürr S 2008 Science $\mathbf{3 2 0} 1329$

[10] Moiseyev N and Cederbaum L S 2005 Phys. Rev. A 72033605

[11] Schlagheck P and Paul T 2006 Phys. Rev. A 73023619

[12] Witthaut D, Graefe E M, Wimberger S and Korsch H J 2006 Phys. Rev. A 75013617

[13] Livi R, Franzosi R and Oppo G L 2006 Phys. Rev. Lett. 97060401

[14] Hiller M, Kottos T and Ossipov A 2006 Phys. Rev. A 73063625 
[15] Schlagheck P and Wimberger S 2007 Appl. Phys. B 86385

[16] Graefe E M, Korsch H J and Niederle A 2008 Preprint arXiv:0807.1777

[17] Dalibard J, Castin Y and Mølmer K 1992 Phys. Rev. Lett. 68580

[18] Carmichael H J 1993 An Open Systems Approach to Quantum Optics (Springer, Berlin)

[19] Albiez M, Gati R, Fölling J, Hunsmann S, Cristiani M and Oberthaler M K 2005 Phys. Rev. Lett. 95010402

[20] Gati R, Hemmerling B, Fölling F, Albiez M and Oberthaler M K 2006 Phys. Rev. Lett. 96 130404; Gati R, Esteve J, Hemmerling B, Ottenstein T B, Appmeier J, Weller A and Oberthaler M K 2006 New J. Phys. 8189

[21] Schumm T, Hofferberth S, Andersson L M, Wildermuth S, Groth S, Bar-Joseph I, Schmiedmayer J and Krüger P 2005 Nature Physics 157

[22] Fölling S, Trotzky S, Cheinet P, Feld M, Saers R, Widera A, Müller T and Bloch I 2007 Nature 4481029

[23] Milburn G J, Corney J, Wright E M and Walls D F 1997 Phys. Rev. A 554318

[24] Smerzi A, Fantoni S, Giovanazzi S and Shenoy S R 1997 Phys. Rev. Lett. 794950

[25] Trimborn F, Witthaut D and Korsch H J 2008 Phys. Rev. A 77 043631; Trimborn F, Witthaut D and Korsch H J 2008 Preprint arXiv:0802.1142

[26] Bloch I, Hänsch T W and Esslinger T 1999 Phys. Rev. Lett. 823008

[27] Gardiner C W and Zoller P 2004 Quantum Noise (Springer Series in Synergetics, Berlin)

[28] Bloch F 1946 Phys. Rev. 70460

[29] Tikhonenkov I, Anglin J R and Vardi A 2007 Phys. Rev. A 75013613

[30] Khodorkovsky Y, Kurizki G and Vardi A 2008 Phys. Rev. Lett. 100220403

[31] Witthaut D, Trimborn F and Wimberger S 2008 in preparation

[32] Rigo M, Alber G, Mota-Furtado F and O'Mahony P F 1997 Phys. Rev. A 551665

[33] Duffield N G and Werner R F 1992 Rev. Math. Phys. 4383 